\title{
A Single State Sliding Mode Controller Design Using Intelligent Optimization Algorithms for the Chaotic WINDMI System
}

\author{
Barış CEVHER, Talha Enes GÜMÜŞ, Mustafa TURAN, Selçuk EMIROĞLU*, Mehmet Ali YALÇIN
}

\begin{abstract}
In this study, a new single-state sliding mode controller is proposed that can be used in multi-input dynamic systems that require the system dynamics to be controlled by a single state variable. The proposed controller is compared with the single state sliding mode controller, which is effective according to a linear feedback controller and a passive controller. To demonstrate the effectiveness of the controllers, the model WINDMI, which is defined as a complex driven damped dynamic system, is preferred as an exemplary model. For the system's dynamics to reach reference in the shortest settling time with minimum overshoot, the sliding surface coefficient and the controller coefficients are optimized according to the sliding surface using Particle Swarm Optimization, Genetic Algorithm, and Pattern Search algorithm. The best values of optimization coefficients are determined by comparing the obtained results. Finally, verifying the effectiveness and reliability of the proposed controller is demonstrated with simulations and numerical analysis.
\end{abstract}

Keywords: Chaos; Chaotic WINDMI System; Intelligent Optimization Algorithms; Sliding Mode Controller

\section{INTRODUCTION}

Chaotic systems are well known to be quite sensitive to initial conditions; each point in the system moves arbitrarily; other points arbitrarily approximate each point in the chaotic system with remarkably different future paths or orbiters. Thus, an arbitrary minor change or perturbation of the current system trajectory may lead to significant differences in system dynamics in the future.

Chaos theory has been a research topic in many scientific disciplines such as engineering, mathematics, physics, meteorology, geology, robotics, computer science, algorithmic trade, philosophy, biology, population dynamics, politics, and finance. These studies have found many application fields and thus become popular in the last thirty years. Some of the application fields and developments in the literature are introduced below.

The researchers have used chaos theory to research and analyze the stability problems in power systems [1-2]. Another application field is secure communication. The chaotic synchronization and its applications in secure communications are studied and suggested a new approach for image encryption based on the chaotic system by many researchers [3-6].

Moreover, the researchers proposed several control methods for controlling, synchronization, and antisynchronization of different chaotic systems such as backstepping control [7-10], feedback control [7-9], the sliding mode control (SMC) [11-23], passive control [23], predictive control [24], synergetic control [25-27], and the internal model control [28-30]. Among these control methods, some of the studies conducted are described below.

In [7], Modified Chua's circuit system applied the feedback control. In [8], a backstepping method is used to design tracking and synchronization controllers. In [9], a modified coupled dynamo system applied feedback and non-feedback methods to control. In [10], for the global chaos synchronization of Sprott's Jerk Systems is designed a backstepping controller. In [11], the chaotic system is controlled via the SMC method. In [12], a Sliding mode controller is suggested to mitigate or eliminate chaos behavior, and a new chaotic system is defined. In [13],
SMC for uncertain stochastic neutral systems with multiple delays is investigated. In [14], a robust adaptive SMC strategy is applied for an introduced class of uncertain chaotic systems. In [15], the SMC technique is implemented to design a power system stabilizer. In [16], Stabilizing unstable periodic orbits of a deterministic chaotic system is studied. In [23], a single state SM controller is shown effectiveness according to the single state linear feedback and the single state passive controller in the hyperchaotic Rabinovich system. In [24], the synchronization of two identical discrete-time chaotic systems is provided with mixed-objective dynamic output feedback robust model predictive control (OFRMPC). In [25], Synergetic Control and synchronization of two different chaotic systems are presented. In [30], the robust control of Chua's circuit underchaos is provided with the internal model principle.

The proper determination and optimal value of the controller coefficients are of great importance. Because it affects the system dynamics in the applications of control, synchronization, and anti-synchronization of chaotic systems, it is challenging to determine or calculate these coefficients in many control approaches. Optimization of these coefficients is another problem [31-33] at the same time. For this purpose, researchers have developed several intelligent optimization algorithms such as Particle Swarm Optimization (PSO) [35, 36], Genetic Algorithm (GA) [37, 38], and Pattern Search (PS) [39].

In this study, the single-state SM controller is proposed for the chaotic WINDMI system. The proposed controller is designed with optimized sliding surface coefficient and controller coefficients by using PSO, GA, and PS. In the second section, the mathematical equations of the chaotic WINDMI system are defined. And the system's parameters and the simulation diagrams of the chaotic WINDMI system without control are also given. In the third section, the design of the single state sliding mode controllers is presented. In the fourth section, the optimization of the sliding surface and controller coefficients with PSO, GA, and PS is described. In the fifth section, numerical simulations on control of the chaotic WINDMI system are performed, and the single-state SM controllers are compared. Finally, conclusions are presented. 


\section{THE WINDMI SYSTEM DESCRIPTION}

The WINDMI model, defined as a complex drivendamped dynamical system, is an energy transfer simulating model based on a physics network between magnetosphere and ionosphere's nightside components (constituents) where the plasma energy is most stored [40-43]. This WINDMI model defined by six-dimensional state space is described by Horton and Doxas in 1996 [40]. A new simplified three-dimensional WINDMI model is introduced by Horton et al. [40-43].

The mathematical model of the chaotic WINDMI system [40-43] is described as given below:

$\frac{\mathrm{d} x_{1}}{\mathrm{~d} t}=x_{2}$

$\frac{\mathrm{d} x_{2}}{\mathrm{~d} t}=x_{3}$

$\frac{\mathrm{d} x_{3}}{\mathrm{~d} t}=-\sigma x_{3}-x_{2}+b-e^{x_{1}}$

For the values of $\sigma=0.7$ and $b=2.5$, the WINDMI system without control becomes a chaotic system, as shown in Fig. 1 and Fig. 2.

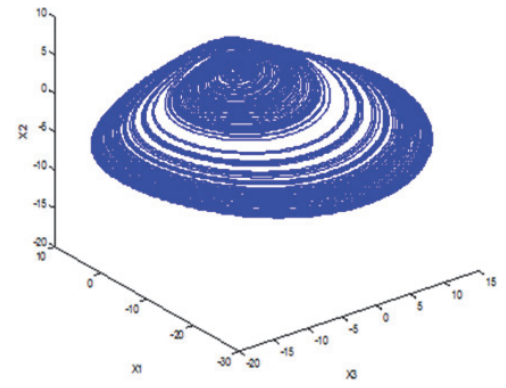

Figure $13-D$ Chaotic phase portrait of the WINDMI system
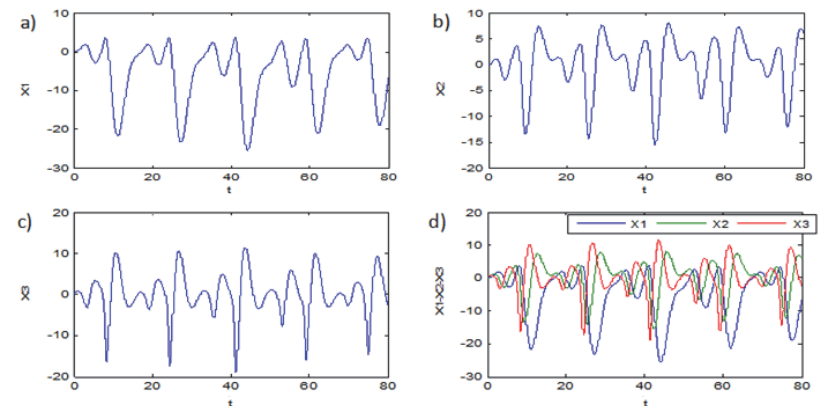

Figure 2 Time series of the uncontrolled WINDMI system a) $x_{1}-t$, b) $x_{2}-t$ c) $x_{3}$ $-t$, d) $x_{1} x_{2} x_{3}-t$

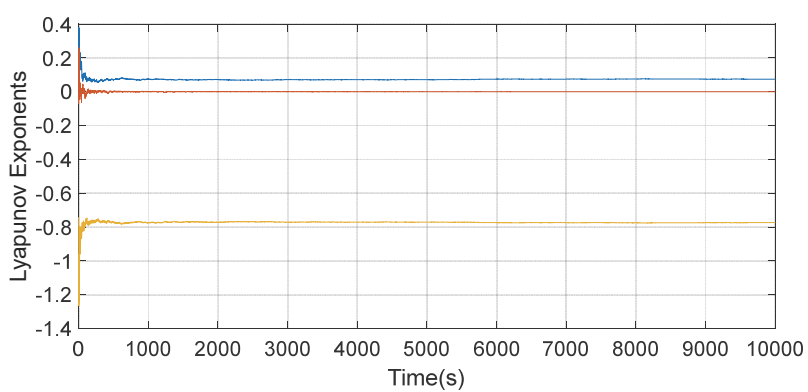

Figure 3 Lyapunov Exponents of the WINDMI chaotic system (0.0737, 0, $-0.7737)$

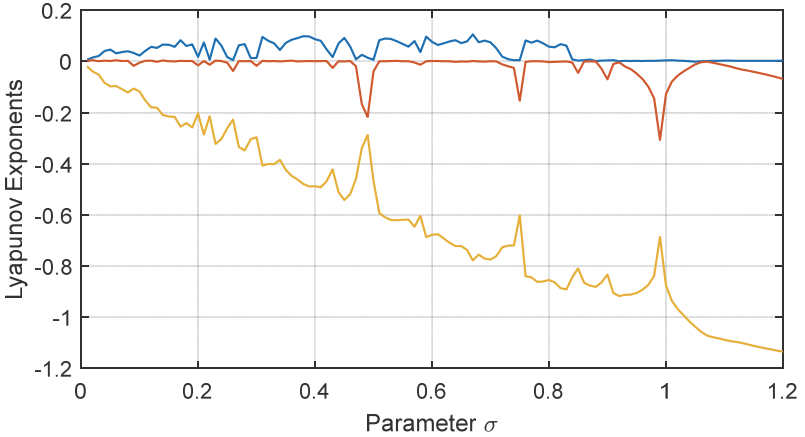

Figure 4 Lyapunov exponent spectrum with respect to $\sigma$

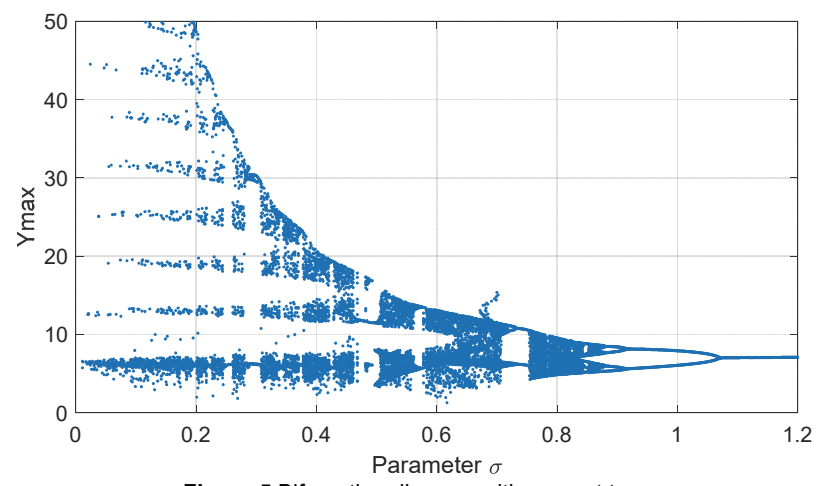

Figure 5 Bifurcation diagram with respect to $\sigma$

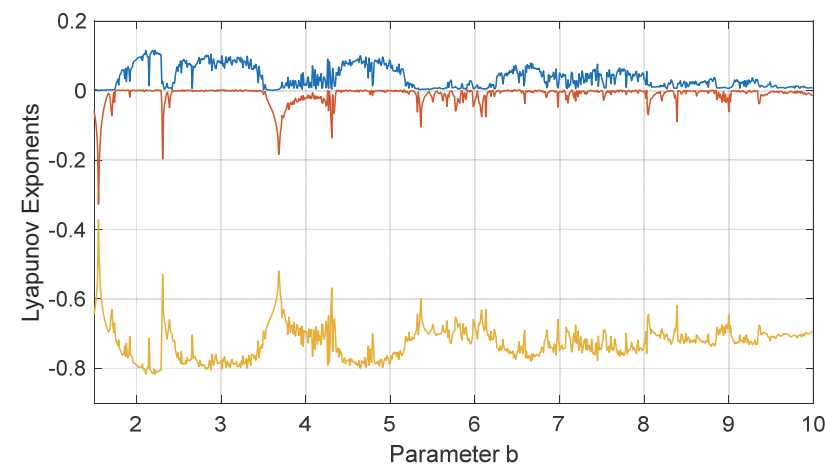

Figure 6 Lyapunov exponent spectrum with respect to $b$

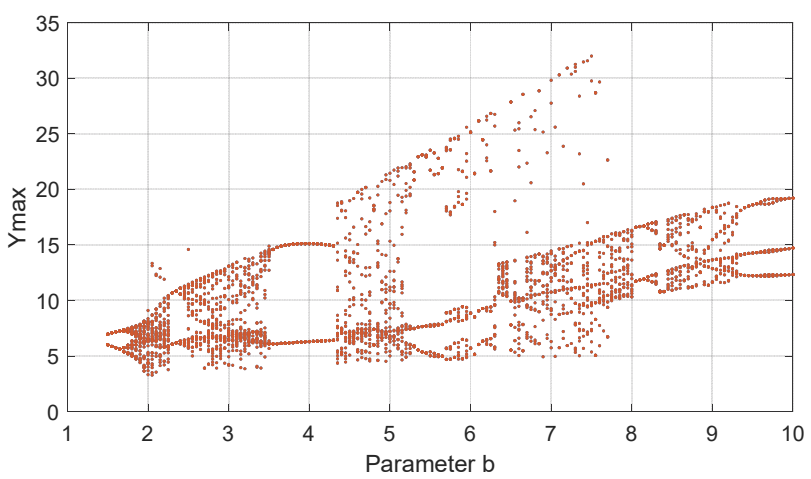

Figure 7 Bifurcation diagram with respect to $b$

To find an equilibrium point of the system, the following Eq. (2) needs to be solved.

$$
\begin{aligned}
& x_{2}=0 \\
& x_{3}=0 \\
& -\sigma x_{3}-x_{2}+b-e^{x_{1}}=0
\end{aligned}
$$


The 3-dimensional WINDMI chaotic system has only one equilibrium at $(0.9163,0,0)$ for $b=2.5$. Using Jacobian and characteristic equation at equilibrium point $(0.9163,0,0)$, the equilibrium point obviously is unstable due to positive eigenvalues $(-1.3424,0.3212+1.3263 i$ $0.3212-1.3263 i)$. As it can be seen from Fig. 3, the Lyapunov exponents of the system are calculated as $L_{1}=$ $0.0737, L_{2}=0$, and $L_{3}=-0.7737$ with parameters $\sigma=0.7$, $b=2.5$, and initial points $x=\left[\begin{array}{lll}0 & 0 & 0\end{array}\right]$ for $T=10^{5} \mathrm{~s}$ using Wolf's algorithm [44]. For $L_{1}>0, L_{1}+L_{2}+L_{3}=-0.7$. So, the sum of Lyapunov exponents is less than zero. KaplanYorke dimension [45] is calculated as follows:

$D_{L}=j+\frac{1}{\left|L_{j+1}\right|} \sum_{i=0}^{j} L_{i}=2+\frac{L_{1}+L_{2}}{\left|L_{3}\right|}=2.0953$ [46]:

The dissipation of the system (1) is given as follows

$\nabla V=\frac{\partial \dot{x}_{1}}{\partial x_{1}}+\frac{\partial \dot{x}_{2}}{\partial x_{2}}+\frac{\partial \dot{x}_{3}}{\partial x_{3}}=-\sigma=-0.7<0$

The divergence is always less than zero due to parameter $\sigma$ being positive. Therefore, the system (1) is clearly dissipative with the rate of an exponential contraction:

$\frac{\mathrm{d} V}{\mathrm{~d} t}=e^{-0.7 t}$

Bifurcation diagram and Lyapunov exponent spectra by changing parameter $\sigma$ and $b$ in $\left(\begin{array}{lll}0 & 1.2\end{array}\right)$ and $\left(\begin{array}{ll}1.5 & 10)\end{array}\right)$ are shown in Fig. 4, Fig. 5, Fig. 6, and Fig. 7, respectively. The parameters $\sigma$ and $b$ in the system (1) change the system's dynamics, as shown in Fig. 6 and Fig. 8 due to the positive Lyapunov exponent. It is known that the system behaves chaotic when the three Lyapunov exponents of the 3D system are positive, zero, and negative. One can deduce from Fig. 4, Fig. 5, Fig. 6, and Fig. 7 in which region and parameter values the system behaves chaotic according to the $\sigma$ and $b$ parameters.

\section{DESIGN OF A SINGLE STATE SLIDING MODE CONTROLLER}

Researchers use many control theories to model the system uncertainties and external distortion in the control, synchronization, and anti-synchronization of the chaotic system. The sliding mode control theory still maintains its popularity among these control theories due to its design flexibility in the sliding surface and switching approach.

SMC method consists of two parts. First, a sliding surface manifold is selected to control the system's trajectory and the system dynamics on the sliding surface in a subsequent time. Second, the controller is designed such that the control signals would reach the manifold and remain on this manifold. As a result, the system reaches the desired dynamic behavior after a limited time.

Let consider the following general equation of an ndimensional chaotic system:

$$
\dot{x}=A x+g+B u
$$

where $A, B, g$, and $u$ are the coefficients of the system state variables, the control matrix, the parameters defining nonlinear uncertainties in the system, and the control inputs, respectively.

The tracking error vector is defined as follows.

$e=x-x_{r e f}$

where $e=\left[e_{1} e_{2} \ldots e_{n}\right]^{\mathrm{T}}$ is described. The error dynamics can be rewritten as follows.

$$
\begin{aligned}
& \dot{e}=\dot{x}-\dot{x}_{r e f} \\
& \dot{e}=A x+g+B u-\dot{x}_{r e f}
\end{aligned}
$$

where $x=\left[\begin{array}{lll}x_{1} & x_{2} & x_{3}\end{array}\right]^{\mathrm{T}}$, is the state variable vector and $x_{\text {ref }}=\left[\begin{array}{lll}x_{1 \text { ref }} & x_{2 \text { ref }} & x_{3 r e f}\end{array}\right]^{\mathrm{T}}$ is the reference state vector.

Now, the sliding surface $s \in R^{3}$ is determined by:

$s(e)=C e$

where the sliding surface is expressed as the scalar equation $s=s(e)$ and $C$ is a constant vector including error dynamics. When the condition below is met, it can be said that the control of the system is achieved $[17,18]$ :

$s(e)=\dot{s}(e)=0$

It should be noted that the necessary condition for the state orbit to remain on the sliding surface is $s(e)=0$. Eq. (9) and Eq. (10) are substituted in Eq. (11) and it can be rewritten as:

$\dot{s}(e)=C A x+C g+C B u-C \dot{x}_{r e f}=0$

Eq. (8) is solved for $u(t)$ by using equivalent control method. The equivalent control $u_{e q}(t)$ is obtained as:

$u_{e q}=-(C B)^{-1}\left[C\left(A x+g-\dot{x}_{r e f}\right)\right]$

Then, the single state SM controller based on Lyapunov and switching theory is designed by using sliding mode [16] and variable structure control theory [15]. By using the constant plus proportional rate reaching law [14] in the single state SM controller, it yields:

$\dot{s}=-K_{d} \operatorname{sgn}(s)-K_{c} s$

$u(t)$ can be obtained as:

$u=-(C B)^{-1}\left[C\left(A x+g-\dot{x}_{r e f}\right)\right]-K_{d} \operatorname{sgn}(s)-K_{c} s$ 
where $K_{c}$ and $K_{d}$ are controller coefficient parameters. The 'sign' is a function as defined below:

$$
\operatorname{sign}(s)=\left\{\begin{array}{r}
1 \text { if } s>0 \\
0 \text { if } s=0 \\
-1 \text { if } s<0
\end{array}\right.
$$

The designed control signal (15) ensures that system dynamics (6) is kept at the sliding surface $s=0$.

Let consider the Lyapunov function as:

$V=\left(s^{2}\right) / 2$

then time derivative along (2) becomes:

$$
\dot{V}=s \dot{s}=-K_{c} s^{2}-K_{d} \operatorname{ssign}(s)
$$

Since $\quad \operatorname{sigign}(s)=|s| \geq 0 \quad$ and $\quad s^{2} \geq 0, \dot{V} \leq 0 \quad$ when $K_{c}>0$ and $K_{d}>0$. Satisfying these conditions allows one to be sure that the designed sliding surface $s$ would asymptotically stabilize the system at the reference point. Achieving this criterion allows to complete the design of the single state SM controller of chaotic system.

\section{OPTIMIZATION OF THE SLIDING SURFACE AND CONTROLLER COEFFICIENTS WITH INTELLIGENCE OPTIMIZATION ALGORITHMS}

Control methods aim to bring the system from the initial state to a desirable state by providing certain quality and performance standards. This can be achieved by control signals affecting the system variables [31]. Thus, the proper control algorithm should be used, and the controller parameters should be selected or calculated properly to control the system and increase the controller's performance. Additionally, optimal choices of the control coefficients are very important to ensure the stability and desirable operation of the control methodology [31].

Thus, optimization methods have been used to increase the performance of the controller or control system because the controller parameters greatly affect the controller performance or output of the system $[32,33]$.

In this study, optimal control parameters of the sliding mode controller have been computed by using intelligent algorithms, which show great performance and are commonly used in optimization problems. The values of sliding surface and controller coefficients have been determined with the help of intelligent algorithms, which are implemented in MATLAB $^{\circledR} /$ SIMULINK $^{\circledR}$ [34]. The sliding mode controller coefficients are optimized by using heuristic optimization methods, GA [35, 36], PSO [37, 38], and pattern search [39]. Algorithms are applied to optimize the sliding mode controller parameters. The heuristic optimization methods have also been used to avoid local optimums. The system with controller coefficients optimized by intelligent algorithms shows better performance than those not optimized with intelligent algorithms.

The controller parameters found by all algorithms under test (PSO, GA, PS) can stabilize the system to the equilibrium or desired point; in other words, the steadystate error becomes zero for all conditions. However, the controller parameters found by PSO give the best results, showing the fast settling time and the lowest overshooting among these intelligent algorithms for the WINDMI system.

To obtain the optimum controller parameters, the integral of absolute error times time (ITEA), which is widely used in the studies, is used as an objective function as below:

$$
\text { ITEA }=\int_{0}^{T} t|e(t)| \mathrm{d} t
$$

$$
e(t)=x(t)-x_{r e f}(t)
$$

where, $x$ and $x_{\text {ref }}$ are the state variables and the references, respectively. $T$ is the time limit of ITEA.

By optimizing the ITAE function [47] given above $(19,20)$, optimum values of the controller parameters are obtained by using the heuristic optimization algorithms. Fig. 8 shows the control scheme of the system with intelligent optimization algorithms. The sliding mode controller optimized with the help of PSO, GA, PS minimizes the settling time and overshoot, as shown in Fig. 9, Fig. 10 and Tab. 1.

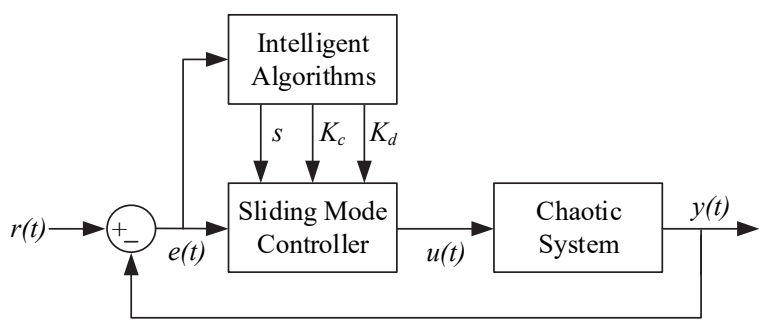

Figure 8 Control scheme of the chaotic WINDMI system based on intelligent algorithms

Table 1 The optimized coefficients for the sliding surface and the single state SM controllers

\begin{tabular}{|c|c|c|c|c|c|c|}
\hline Controllers & Algorithm & Sliding Surface & $K_{c}$ & $K_{d}$ & Objective $(I T E A)$ & Settling time \\
\hline & NO & $\gamma=1$ & 0.4 & 0.5 & 2125.95 \\
\cline { 2 - 6 } Proposed SM controller & PSO & $\gamma=1.4958$ & 0.5262 & 0.9499 & 2100.83 \\
\cline { 2 - 6 } & GA & $\gamma=1.4348$ & 0.5256 & 0.8420 & 2103.65 \\
\cline { 2 - 6 } & PS & $\gamma=1.5565$ & 0.5409 & 0.9917 & 2101.04 \\
\hline Compared SM controller & PSO & $k_{1}=0.9842$ & 0.5262 & 0.8420 & 2172.72 \\
\hline
\end{tabular}

The proposed algorithm based on heuristic optimization has been run several times to avoid local optimums and ensure the best solution. The optimized controller parameters that make the objective function the minimum are obtained by using intelligent algorithms. The controller parameters optimized by PSO, GA, PS heuristic 
algorithms and the settling time of the system showing controller performance are given in Tab. 1 .

As shown in Tab. 1, the settling time obtained by PSO is the smallest among the algorithms used in implementation; it is observed that the objective function (ITEA) value is the smallest one.

\section{THE SINGLE STATE SM CONTROLLERS COMPARED FOR THE CHAOTIC WINDMI SYSTEM BY NUMERICAL SIMULATIONS}

The mathematical model of the controlled chaotic WINDMI system is described as follows:

$$
\begin{aligned}
\frac{\mathrm{d} x_{1}}{\mathrm{~d} t} & =x_{2} \\
\frac{\mathrm{d} x_{2}}{\mathrm{~d} t} & =x_{3} \\
\frac{\mathrm{d} x_{3}}{\mathrm{~d} t} & =-\sigma x_{3}-x_{2}+b-e^{x_{1}}+u
\end{aligned}
$$

$$
A=\left[\begin{array}{ccc}
0 & 1 & 0 \\
0 & 0 & 1 \\
0 & -1 & -\sigma
\end{array}\right], B=\left[\begin{array}{l}
0 \\
0 \\
1
\end{array}\right], g=\left[\begin{array}{c}
0 \\
0 \\
b-e^{x_{1}}
\end{array}\right]
$$

The error dynamics coefficients $C_{1}$ and $C_{2}$ are selected for the proposed SM controller and the compared SM controller [23] respectively as given below:

$$
C_{1}=\left[\begin{array}{lll}
\gamma & 1 & 1
\end{array}\right] \text { and } C_{2}=\left[\begin{array}{lll}
0 & k_{1} & 1
\end{array}\right]
$$

The sliding surfaces are determined as given below:

$$
\begin{aligned}
& s_{1}=\gamma e_{1}+e_{2}+e_{3} \\
& s_{2}=k_{1} e_{2}+e_{3}
\end{aligned}
$$

where $s_{1}$ and $s_{2}$ are the sliding surfaces for the proposed SM controller and the compared SM controller [23] respectively. $\gamma, k_{1}$ are the error dynamics coefficients and $e=x-x_{r e f}$ is the tracking error vector.

As the second step, reaching mode control law which holds the system trajectories onto the sliding surface $(s=$ 0 ) is determined [18]. In this study, the reaching law is defined by Eq. (23):

$$
\dot{s}=-K_{c} s-K_{d} \operatorname{sign}(s)
$$

where $K_{c}$ and $K_{d}$ are the controller coefficients and are the optimized design parameters.

By making the derivative of sliding surfaces and reachability condition equal, the control signals $u_{1}$ and $u_{2}$ are obtained respectively as given below:

$$
\begin{aligned}
u_{1}= & -K_{c} s-K_{d} \operatorname{sign}(s)+x_{2}(1-\gamma)-x_{3}(1-\sigma) \\
& -b+e^{x_{1}}+\gamma \dot{x}_{1 r e f}+\dot{x}_{2 r e f}+\dot{x}_{3 r e f} \\
u_{2}= & -K_{c} s-K_{d} \operatorname{sign}(s)+e_{2}-e_{3}\left(\sigma-k_{1}\right)-b+e^{e_{1}}
\end{aligned}
$$

Thus, the design of the single state SM controllers is completed for control of the chaotic WINDMI system. The designed control signals (24) ensure that the system dynamics (21) is kept onto the sliding surface $s=0$.

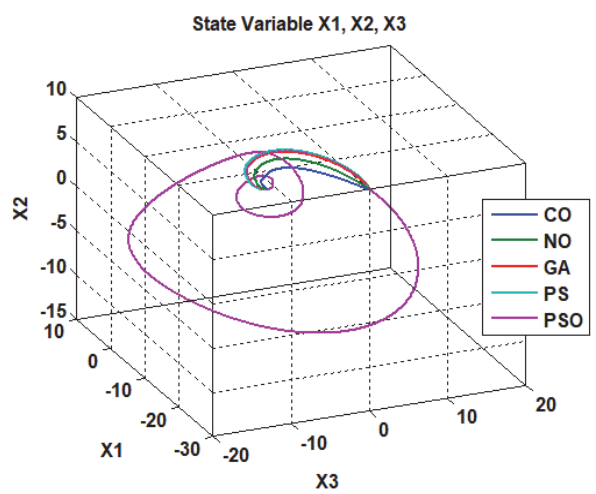

Figure $93 \mathrm{D}$ phase portrait of the chaotic WINDMI system, controlled by the activation of the SMC control signals after $12 \mathrm{~s}$.

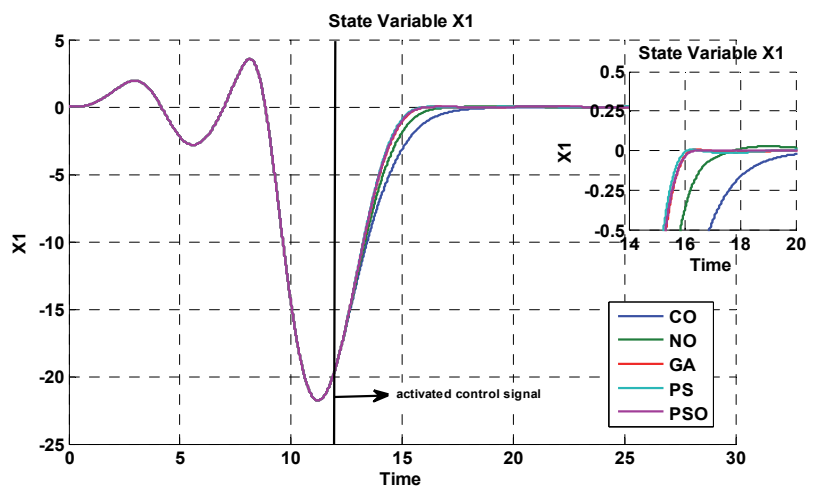

a)

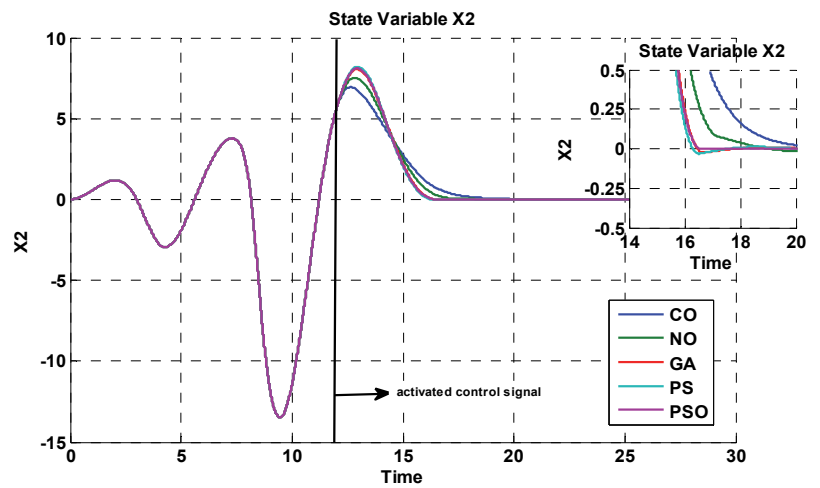

b)

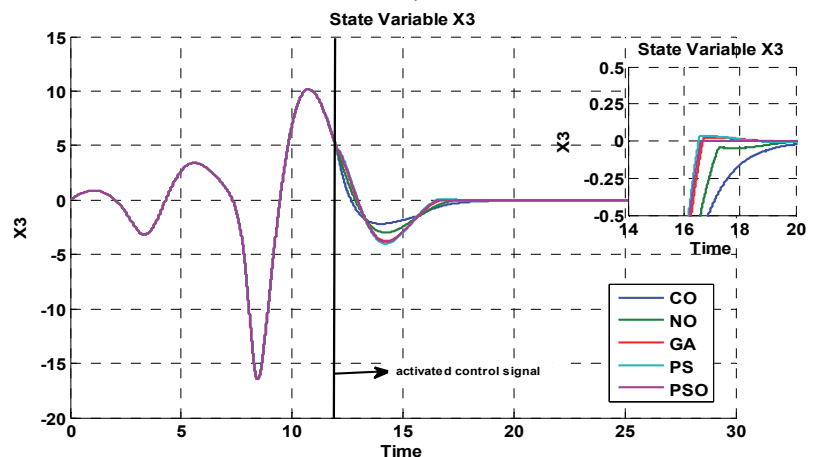

c)

Figure 10Time series of the WINDMI System with SMC after $12 \mathrm{~s}$. a) $x_{1}-t$, b) $x_{2}-t$ c) $x_{3}-t$

The parameters defining the chaotic WINDMI system are $\sigma=0.7, b=2.5$, the initial condition $x_{0}=\left[\begin{array}{lll}0 & 0 & 0\end{array}\right]$ and the 
step size is 0.0001 . After applying the control signals to the chaotic WINDMI system at $12 \mathrm{~s}$, the system's trajectory reaches a stable state. The system converges to reference point $x_{\text {ref }}=\left[\begin{array}{lll}0 & 0 & 0\end{array}\right]$ as shown in Fig. 9. In addition, the optimal values of the controller coefficients and the optimal value of the sliding surface's tuning parameter were determined by using intelligent optimization algorithms to increase the efficiency of the controllers. With the control signals obtained using the determined coefficients, the trajectory of the system's state variables reaching a stable point is shown with colored lines in Fig. 9. For better visibility of the results achieved, the time series of state variables are shown in Fig. 10.

It can be seen from Fig. 9 and Fig. 10, the designed and optimized single-state SM controllers stabilize the chaotic WINDMI system successfully. The single-state SM controllers optimized by the PSO algorithm give almost no overshoot. In addition, the settling time is the smallest for the PSO algorithm, as shown in Fig. 10. Although the proposed controller optimized by GA and PS provides zero steady-state error, overshoot occurs.

It should be noted that the proposed SM controllers optimized and non-optimized by GA-PS-PSO are named NO and GA-PS-PSO and, the compared SM controller optimized by PSO is named CO in Fig. 10, respectively.

\section{CONCLUSION}

In this study, a single state SM controller in which the sliding surface coefficient and the controller coefficients are optimized according to the sliding surface is proposed for the system's dynamics to reach reference in the shortest settling time with minimum overshoot. The proposed controller is compared with the single state SM controller, which is effective according to a linear feedback controller and a passive controller.

The proposed SM controller and the compared SM controller are simulated on the chaotic WINDMI system. Also, to increase the controllers' effectiveness, the optimal values of the sliding surface and controller coefficients are found with intelligent optimization algorithms, and compared. The simulation results showed that the settling time obtained by PSO is the smallest among the algorithms chosen, and the objective function (ITEA) value is the smallest one. Besides, the effectiveness of the proposed single-state SM controller to the compared single-state SM controller is shown on the chaotic WINDMI system. It can be seen from the results obtained that the proposed singlestate SM controller can solve chaotic problems such as the chaotic WINDMI system.

The suggested controller can be applied to control, synchronize and anti-synchronize hyperchaotic systems in further studies.

\section{REFERENCES}

[1] Yu, Y., Jia, H., \& Li, J., (2003) Power system instability and chaos, Electric Power Systems Research, 65(3), 187-195. https://doi.org/10.1016/S0378-7796(02)00229-8

[2] Rajesh, K. G. \& Padiyar, K. R. (1999). Bifurcation analysis of a three-node power system with detailed models, International Journal of Electrical Power \& Energy Systems., 21(5), 375-393.

https://doi.org/10.1016/S0142-0615(99)00002-2
[3] Yeh, J. P. \& Wu, K. L. (2008). A simple method to synchronize chaotic systems and its application to secure communications. Mathematical and Computer Modeling, 47(9-10), 894-902. https://doi.org/10.1016/j.mcm.2007.06.021

[4] Lin, Z. \& Wang, H. (2010). Efficient Image Encryption using a Chaos-based PWL Memristor. IETE Technical Review, 27(4), 318-325. https://doi.org/10.4103/0256-4602.64605

[5] Pareek, N. K., Patidar, V., \& Sud, K. K. (2006). Image encryption using chaotic logistic map. Elsevier Image and Vision Computing, 24, 926-934. https://doi.org/10.1016/j.imavis.2006.02.021

[6] Mandal, M. K., Banik, G. D., Chattopadhyay, D., \& Nandi, D. (2012). An image encryption process based on Chaotic logistic map. IETE Technical Review, 29(5), 395-404. https://doi.org/10.4103/0256-4602.103173

[7] Wu, T., \& Chen, M. S.(2002). Chaos control of the modified Chua's circuit system, Physica D, 164, 53-58. https://doi.org/10.1016/S0167-2789(02)00360-3

[8] Wang, X. Y. \& Zhang, J. (2011). Tracking Control and The Backstepping Design of Synchronization Controller for Chen System. International Journal of Modern Physics B. 25(28), 3815-3824. https://doi.org/10.1142/S0217979211059073

[9] Wang X. Y. \& Xiang, J. W. (2007). Chaos Control of a Modified Coupled Dynamos System. International Journal of Modern Physics B, 21(26), 4593-4610. https://doi.org/10.1142/S0217979207037818

[10] Vaidyanathan, S., Idowu, B. A., \& Azar, A. T. (2015). Backstepping Controller Design for the Global Chaos Synchronization of Sprott's Jerk Systems. Chaos Modeling and Control Systems Design, Springer, Switzerland, 39-58. https://doi.org/10.1007/978-3-319-13132-0_3

[11] Harb, A. M. \& Natsheh, A. N. (2009). On sliding-mode control of chaotic systems. International Journal of Modeling and Simulation, 29(1), 89-95. https://doi.org/10.1080/02286203.2009.11442514

[12] Ming, L. \& Chong, X. L.(2010). Sliding mode control of a new chaotic system.Chin. Phys. B, 19(10), 100504-1, 100504-3. https://doi.org/10.1088/1674-1056/19/10/100504

[13] Chen, D. \& Zhang, W. (2008). Sliding Mode Control of Uncertain Neutral Stochastic Systems with Multiple Delays. Mathematical Problems in Engineering, 11. https://doi.org/10.1155/2008/761342

[14] Roopaei, M., Sahraei, B. R., \& Lin, T. C. (2010). Adaptive sliding mode control in a novel class of chaotic systems.Elsevier Com. Nonlinear Sci. Num. Sim., 15, 41584170. https://doi.org/10.1016/j.cnsns.2010.02.017

[15] Shahgholian, G., Rajabi, A., \& Karimi, B. (2010). Analysis and Design of PSS for Multi-Machine Power System Based on Sliding Mode Control Theory. International Review of Electrical Engineering (IREE), 5(5), 2241-2250.

[16] Salarieh, H. \& Alasty, A. (2009). Control of stochastic chaos using sliding mode method. Journal of Computational and Applied Mathematics., 225, 135-145. https://doi.org/10.1016/j.cam.2008.07.032

[17] Eker, I. (2006). Sliding mode control with PID sliding surface and experimental application to an electromechanical plant.ISA Transactions, 45(1), 109-118. https://doi.org/10.1016/S0019-0578(07)60070-6

[18] Hung, J. Y., Gao, W., \& Hung, J. C. (1993). Variable structure control: a survey. IEEE Trans Indust Electron, 40, 2-22. https://doi.org/10.1109/41.184817

[19] Young, K. D., Utkin, V. I., Ozguner, U. (1999). A control engineer's guide to sliding mode control.IEEE Trans Control Systems Tech., 7, 328-342. https://doi.org/10.1109/87.761053

[20] Perruquetti, W. \& Barbot, J. P. (2002). Sliding Mode Control in Engineering, Marcel Dekker. https://doi.org/10.1201/9780203910856 
[21] Wei, Z., Yousefpour, A., Jahanshahi, H., Kocamaz, U. E., \& Moroz, I., (2021). Hopf bifurcation and synchronization of a five-dimensional self-exciting homopolar disc dynamo using a new fuzzy disturbance-observer-based terminal sliding mode control.Journal of the Franklin Institute, 358(1), 814833. https://doi.org/10.1016/j.jfranklin.2020.11.011

[22] Utkin, V. I. (1978). Sliding Mode and Their Application in Variable Structure Systems, Mir Editors, Moscow.

[23] Kocamaz, U. E., Uyaroğlu, Y., \& Kızmaz, H. (2017). Controlling hyperchaotic Rabinovich system with single state controllers: Comparison of linear feedback, sliding mode, and passive control methods, Optic, Elsevier, V. 130, 914-921. https://doi.org/10.1016/j.jileo.2016.11.006

[24] Jiang, W., Wang, H., Lu, J., Cai, G., \& Qin, W. (2017). Synchronization for chaotic systems via mixed-objective dynamic output feedback robust model predictive control, Journal of the Franklin Institute, 354(12), 4838-4860. https://doi.org/10.1016/j.jfranklin.2017.05.007

[25] Harmas, M. N. (2016). Synergetic Control and Synchronisation of Chaotic Systems. International Journal of Control, Automation, Commun. and Syst., 1(2),31-39. https://doi.org/10.5121/ijcacs.2016.1204

[26] Ni, J., Liu, C., Liu, K., \& Pang, X. (2014). Variable speed synergetic control for chaotic oscillation in power system. Nonlinear Dynamics, 78(1), 681-690. https://doi.org/10.1007/s11071-014-1468-0

[27] Liu, C. \& Hsiao, M. (2013). Synchronization on Unified Chaotic Systems via PI-type Terminal Synergetic Control. 2013 CACS International Automatic Control Conference (CACS), Taiwan, 24-29. https://doi.org/10.1109/CACS.2013.6734101

[28] Wei, X., Wang, J., \& Deng, B. (2009). Introducing internal model to robust output synchronization of FitzHughNagumo neurons in external electrical stimulation, Communications in Nonlinear Science and Numerical Simulation, 14(7), 3108-3119. https://doi.org/10.1016/j.cnsns.2008.10.016

[29] Henson, M. A., \& Seborg, D. E. (1991). An internal model control strategy for nonlinear systems. AIChE Journal, 37(7), 1065-1081. https://doi.org/10.1002/aic.690370711

[30] Lee, K. W. \& Singh, S. N. (2007). Robust control of chaos in Chua's circuit based on internal model principle. Chaos, Solitons \& Fractals, 31(5), 1095-1107. https://doi.org/10.1016/j.chaos.2005.10.058

[31] Boccaletti, S., Grebogi, C., Lai, Y. C., Mancini, H., \& Maza, D. (2000). The control of chaos: theory and applications. Elsevier Physics Reports, 329(103), 197-205. https://doi.org/10.1016/S0370-1573(99)00096-4

[32] Dash, P., Chandra S. L., \& Sinha, N. (2015). Automatic generation control of multi area thermal system using Bat algorithm optimized PD-PID cascade controller. Electrical Power and Energy Systems, 68, 364-372. https://doi.org/10.1016/j.ijepes.2014.12.063

[33] Das, S., Acharya, A., \& Pan, I. (2014). Simulation studies on the design of optimum PID controllers to suppress chaotic oscillations in a family of Lorenz-like multi-wing attractors, Mathematics and Computers in Simulation, 100, 72-87. https://doi.org/10.1016/j.matcom.2014.03.002

[34] T. Math Works, "MATLAB (2020), MathWorks Inc., no. x, 2020.

[35] Holland, J. (1975). Adaptation in Natural and Artificial Systems. The University of Michigan Press, Ann Arbor.

[36] Goldberg, D. E. (1989). Genetic Algorithms in Search, Optimization \& Machine Learning. Addison-Wesley.

[37] Kennedy, J. \& Eberhart, R. C. (1995). Particle swarm optimization. Proc. IEEE int'l conf. on neural networks.4, 1942-1948. IEEE service center, Piscataway, NJ. http://dx.doi.org/10.1109/ICNN.1995.488968

[38] Eberhart, R. C. \& Kennedy, J. (1995). A new optimizer using particle swarm theory. Proceedings of the sixth international symposium on micro machine and human science, 39-43. IEEE service center, NJ, Nagoya, Japan. https://doi.org/10.1109/MHS.1995.494215

[39] Al-Othman, K. \& El-Naggar, K. M. (2008). Application of pattern search method to power system security constrained economic dispatch with non-smooth cost function, Electric Power Systems Research, 78,667-675. https://doi.org/10.1016/j.epsr.2007.05.015

[40] Horton, W. \& Doxas, I. (1996). A low dimensional energy conserving state space model for substorm dynamics. $J$. Geophys. Res., 101(A12), 27223-27237. https://doi.org/10.1029/96JA01638

[41] Horton, W., Weigel, R. S., \& Sprott, J. C. (2001). Chaos and the limits of predictability for the solar-wind-driven magnetosphere-ionosphere system. Physics of Plasmas, 8(6), 2946-2952. https://doi.org/10.1063/1.1371522

[42] Horton, W. \& Doxas, I. (1998). A low-dimensional dynamical model for the solar wind driven geotailionosphere system. Journal of Geophysical Research A, 103(A3), 4561-4572. https://doi.org/10.1029/97JA02417

[43] Smith, J. P., Thiffeault, J. L., \& Horton, W. (2000). Dynamical range of the WINDMI model: an exploration of possible magnetospheric plasma states. Journal of Geophysical Research A, 105(A6), 12983-12996. https://doi.org/10.1029/1999JA000218

[44] Wolf, A., Swift, J. B., Swinney, H. L., \& Vastano, J. A. (1985). Determining Lyapunov exponents from a timeseries. Physica D: Nonlinear Phenomena, 16(3), 285-317. https://doi.org/10.1016/0167-2789(85)90011-9

[45] Kuznetsov, N. \& Reitmann, V. (2021). Attractor Dimension Estimates for Dynamical Systems: Theory and Computation of Emergence, Complexity and Computation, Springer International Publishing https://doi.org/10.1007/978-3-030-50987-3

[46] Shivamoggi, B. K. (2014). Chaos in dissipative systems, in Nonlinear Dynamics and Chaotic Phenomena: An Introduction. Fluid Mechanics and Its Applications. 103, Springer, Dordrecht, 189-244. https://doi.org/10.1007/978-94-007-7094-2_6

[47] Davendra, D., Zelinka, I., Senkerik, R. (2010). Chaos driven evolutionary algorithms for the task of PID control. Comput. \& Mathem. with Applications, 60(4), 1088-1104. https://doi.org/10.1016/j.camwa.2010.03.066

\section{Contact information:}

\section{Barış CEVHER}

Sakarya University, Faculty of Engineering, Electrical and Electronics Eng., Esentepe Campus, Serdivan, Sakarya, Turkey

E-mail: bcevher@sakarya.edu.tr

\section{Talha Enes GÜMÜŞ}

Sakarya University, Faculty of Engineering, Electrical and Electronics Eng., Esentepe Campus, Serdivan, Sakarya, Turkey

E-mail: tgumus@sakarya.edu.tr

\section{Mustafa TURAN}

Sakarya University, Faculty of Engineering, Electrical and Electronics Eng., Esentepe Campus, Serdivan, Sakarya, Turkey

E-mail: turan@sakarya.edu.tr

\section{Selçuk EMIROĞLU}

(Corresponding author)

Sakarya University, Faculty of Engineering, Electrical and Electronics Eng., Esentepe Campus, Serdivan, Sakarya, Turkey

E-mail: selcukemiroglu@sakarya.edu.tr

\section{Mehmet Ali YALCIN}

Sakarya University, Faculty of Engineering, Electrical and Electronics Eng., Esentepe Campus, Serdivan, Sakarya, Turkey

E-mail: yalcin@sakarya.edu.tr 